AperTO - Archivio Istituzionale Open Access dell'Università di Torino

The risk of ischaemic stroke in primary APS patients: a prospective study

This is a pre print version of the following article:

Original Citation:

Availability:

This version is available http://hdl.handle.net/2318/1653357

since 2019-01-31T13:23:07Z

Published version:

DOI:10.1111/ene.13499

Terms of use:

Open Access

Anyone can freely access the full text of works made available as "Open Access". Works made available under a Creative Commons license can be used according to the terms and conditions of said license. Use of all other works requires consent of the right holder (author or publisher) if not exempted from copyright protection by the applicable law. 
This is the author's final version of the contribution published as:

Radin Massimo (MD), Schreiber Karen (MD), Cecchi Irene (MD), Roccatello Dario (MD), Cuadrado Maria José (MD, PhD) and Sciascia Savino (MD, PhD).

The risk of ischaemic stroke in primary APS patients: a prospective study. Eur J Neurol. 2018 Feb;25(2):320-325. doi: 10.1111/ene.13499.

The publisher's version is available at:

https://onlinelibrary.wiley.com/doi/abs/10.1111/ene.13499

When citing, please refer to the published version.

Link to this full text:

http://hdl.handle.net/2318/1653357 


\section{The risk of ischaemic stroke in primary APS patients: a prospective study}

Radin Massimo (MD) ${ }^{1}$, Schreiber Karen (MD) ${ }^{2,3}$, Cecchi Irene (MD) ${ }^{1}$, Roccatello Dario (MD) ${ }^{1}$, Cuadrado Maria José (MD, PhD) ${ }^{4}$ and Sciascia Savino (MD, PhD) 1 .

${ }^{1}$ Center of Research of Immunopathology and Rare Diseases- Coordinating Center of Piemonte and Valle d'Aosta Network for Rare Diseases, Department of Clinical and Biological Sciences, and SCDU Nephrology and Dialysis, University of Turin and S. Giovanni BoscoHospital, Turin, Italy.

2Department of Thrombosis and Haemophilia, Guy's and St Thomas' Hospital, London, United Kingdom.

${ }^{3}$ Department of Rheumatology, Copenhagen University Hospital, Copenhagen, Denmark ${ }^{4}$ Louise Coote Lupus Unit, Guy's and St Thomas' NHS Foundation Trust, London, UK.

Cover Title: aPL and risk of stroke in PAPS

Key words: Antiphospholipid syndrome - APS - stroke - GAPSS - risk score - thrombosis - aPL Corresponding Author:

Savino Sciascia, MD, PhD;

Center of Research of Immunopathology and Rare Diseases- Coordinating Center of Piemonte and Valle d'Aosta Network for Rare Diseases, and SCDU Nephrology and Dialysis, S. Giovanni Bosco Hospital

Piazza del Donatore di Sangue 3, 10154, Turin, Italy.

Email savino.sciascia@unito.itTel +390112402056 Fax +390112402052

This author takes responsibility for all aspects of the reliability and freedom from bias of the data presented and their discussed interpretation

Total word count: 2204

Funding: none 


\section{Abstract}

\section{Background:}

The most common neurological manifestation of APS is ischaemic stroke. Identifying patients with APS at high risk for developing any thrombotic event remains a major challenge. In this study, we aimed to identify predictive factors of ischaemic stroke in a cohort of primary APS (PAPS) patients who presented with new onset symptoms suggestive of acute stroke.

\section{Methods:}

This prospective multicenter study included 36 consecutive PAPS patients who presented with new onset symptoms suggestive of an acute stroke. Patients were prospectively followed-up for 12 months.

\section{Results:}

In ten (28\%) out of 36 PAPS patients [mean age 41 years old (S.D.13.4), female (70\%)], the suspicion of an acute stroke was confirmed by brain MRI. Sixty percent of these patients were $<50$ years old. Six out of the ten patients had a history of previous venous thrombosis and were receiving vitamin $\mathrm{K}$ antagonist (VKA), with INR target 2-3; one patient had a history of a previous arterial event receiving treatment with VKA target INR 2-3 plus low dose aspirin (LDA), and one patient had a history of previous pregnancy morbidity receiving only LDA. Time in therapeutic range for patients receiving VKA was 77.7 (S.D.6.6). Hypercholesterolemia was significantly higher in patients with confirmed stroke 
when compared to those without $(\mathrm{p}<0.05)$. Similarly, we found a significantly higher rate of anti-ß2GPI antibodies $(\operatorname{IgG} / \operatorname{IgM})(\mathrm{p}<0.05)$ and higher aGAPSS values in patients with a confirmed stroke [mean aGAPSS 8.9(S.D.4.7) Vs. mean aGAPSS6.4(S.D.2.5);p:<0.05].

\section{Conclusions:}

Patients with PAPS, including young patients, have a high risk of recurrent thrombosis despite anticoagulation treatment. A careful risk assessment is mandatory to identify patients at risk for recurrence. 


\subsection{Introduction}

The antiphospholipid syndrome (APS), recognized as the most common acquired thrombophilia, is an autoimmune condition characterized by the occurrence of thrombotic events and/or pregnancy morbidity in individuals found to be persistently positive for antiphospholipid antibodies (aPL) [1]. The current classification criteria for APS include three laboratory tests: lupus anticoagulant (LA), anti-cardiolipin (aCL), and anti- $\beta 2$ glycoprotein-I ( $\beta 2 \mathrm{GPI}$ ) antibodies. To prevent the detection of transient antibodies, tests must be positive on two separate occasions, at least 12 weeks apart [1]. APS can be associated with another autoimmune disease, mainly systemic lupus erythematosus, and has in the past been described as 'secondary' APS. On the other hand, APS can be found as an isolated condition called 'primary' APS (PAPS).

Thrombotic events in APS can affect the venous and the arterial system [1]. While deep vein thrombosis represents the most frequent thrombotic feature of APS, arterial events include the potentially most life-threatening manifestations of the syndrome, being the central nervous system the most common affected site $[2,3]$. Ischaemic stroke involving the territory of the middle cerebral artery is the main neurological manifestation associated with APS [2]. However, transient ischaemic attack (TIA), amaurosisfugax, transient paresthesias, 
cerebral venous sinus thrombosis, ocular ischaemia, and acute ischaemic encephalopathy have been also described $[2,4]$.

Considering all patients with cerebral ischaemic events, the prevalence of aPL in young adults below the age of 50 has been reported as high as $17.2 \%$ (range 2\%-56\%)[5]. Given their young age and how the development of cerebrovascular events can negatively influence the quality of life, patients with aPL positivity represents a particular subgroup at greater risk of developing serious stroke and in which the development of risk stratification models is necessary [5].

To date, identifying patients with aPL positivity who are at high risk for developing any thrombotic event, including cerebrovascular manifestations, is still an unmet clinical need and remains a major challenge for the treating physicians.

Recently, our group developed a risk score for clinical manifestations of APS [the global APS score [GAPSS)] that takes into account the combination of conventional risk factors for cardiovascular events and the aPL profile[6]. In this study, we aimed to prospectively identify predictive factors of ischaemic stroke in a cohort of PAPS patients with a clinical suspicion of acute stroke event and to further evaluate the rate of recurrence and/or progression of cerebral lesions and potential new subclinical signs of infarction after 12 months of follow-up. 


\subsection{Patients and methods:}

\subsection{Patients}

This prospective multicenter study included 36 consecutive patients with known history of PAPS (either previous arterial or venous thrombotic events or pregnancy morbidity) attending the Giovanni Bosco Hospital, Turin, Italy and the Louise Coote Lupus Unit at St Thomas' Hospital, London, UK who presented with a new episode clinically suggestive of an acute stroke to our departments or the emergency departments within our hospitals (enrollment time January 2015-February 2017). The decision to conduct brain imaging was initiated by the emergency physician and it was based on the suspicion of an acute stroke. Every patient was additionally clinically assessed by a neurologist as per standard of care in our two centers.

Study inclusion criteria comprehend: a) the diagnosis of PAPS according to Sidney criteria [1], b) the onset signs and/or symptoms of new focal neurological deficits suggestive of an acute stroke evaluated by a neurologist.

The indication for performing the MRI was the suspicion of an acute cerebrovascular event following the National Institute for Health and Care Excellence (NICE) guidelines based on the onset of new focal neurological deficits [7]. The presence of concomitant or any previous history of headaches (including migraines with and without aura), cognitive impairment, and self- 
reported word finding difficulties were recorded. The assessment for cerebral events included brain computed tomography, MRI, carotid ultrasound, electrocardiogram, and echocardiography. Imaging findings were discussed in multidisciplinary meetings.

At admission, patients in the stroke group were screened for alternative etiologies (e.g. substance abuse, carotid/vertebral artery dissections and cardioembolic etiology). In detail, after an initial brain MRI to confirm the ischeamic event, neck and cerebral vascular imaging (e.g., CTA for head and neck), transthoracic echocardiogram with a bubble study, additional risk factor blood work (e.g., TSH, ESR, CRP, HIV, and toxicology screen), and, when appropriate, sickle screen and pregnancy test where performed according treating physician's judgment.

Patients were followed-up for 12 months. A follow-up cerebral MRI was performed after 12 months in all patients to assess the outcome of the abnormal findings reported in the first brain MRI and to investigate potential new subclinical signs of infarction.

Demographic, clinical and laboratory characteristics are summarized in Table 1.

\subsection{Cardiovascular risk factors assessment}

Cardiovascular risk factors of the study population were assessed following the NICE guidelines [8]. In detail, enrolled patients underwent a physical examination, blood pressure determination, and phlebotomy in order to assess 
vascular risk factors. Arterial hypertension was defined as an appropriately sized cut-off [8], high blood pressure on at least two occasions, and/or use of oral antihypertensive medications. Serum total and high-density lipoprotein cholesterol levels were determined according to standardized methods and interpreted according to current cut-off values [8].

\section{Autoantibody detection}

The aPL profile included LA, aCL, and anti-ß2GPI antibodies. The aCL and antiß2GPI were detected by ELISA as described previously $[9,10]$. Plasma samples were tested for the presence of LA according to the recommended criteria from the International Society on Thrombosis and Haemostasis (ISTH) Subcommittee on Lupus Anticoagulant/Phospholipid-Dependent Antibodies [11,12]

\subsection{Adjusted GAPSS calculation}

The cumulative aGAPSS was calculated for each patient as previously reported, by adding together all points corresponding to the risk factors [6,13]. After its first description, GAPSS was prospectively validated [14] and applied in a cohort of patients with PAPS [15]. To increase the generalizability of the findings, in this study we applied an adjusted version of GAPSS (aGAPSS). This comprises only aPL testing included in the current classification criteria for APS (excluding aPSPT, not routinely available in all the laboratories). Data are presented as aGAPSS. 
For the purpose of this study, all computed variables refer to values/parameters assessed at the time of the suspected stroke and after 12 months of follow up.

\subsection{Statistical analysis}

Categorical variables are presented as number (\%) and continuous variables are presented as mean (S.D.). The significance of baseline differences was determined by the chi-squared test, Fisher's exact test or the unpaired t-test, as appropriate. A two-sided P-value $<0.05$ was statistically significant. All statistical analyses were performed using SPSS version 19.0 (IBM, Armonk, NY, USA).

\subsection{Results:}

A total of 36 consecutive PAPS patients [mean age 32 years old (SD 33.7), female $(86 \%)]$ with suspect stroke were included in this prospective study. Twenty-five patients $(69 \%)$ were $<50$ years old at the time of the first event. All patients fulfilled the current classification criteria for PAPS.

New focal neurological deficits were distributed as follows: six with dysphasia or dysarthria, four with self-reported speech disturbance, eight with subjective arm or leg weakness, 13 with subjective arm or leg paresthesia and 16 with new onset of severe headache.

\subsection{Ischaemic Stroke}


In ten patients (28\%) [mean age 41 years old (SD 13.4), female (70\%)] the suspicion of an acute ischaemic stroke was confirmed and MRI findings were compatible with a new ischaemic lesion. Demographics, cardiovascular risk factors, and aPL profile at the time of the stroke are summarized in Table 2. In the remaining 26 patients, the diagnosis of ischaemic stroke was ruled out after clinical and imaging evaluation.

When analyzing for cardiovascular risk factors, hypercholesterolemia was significantly higher in the patients with confirmed stroke when compared to those without (Chi square test: $\mathrm{p}<0.05$ ). No evidence of clinical significant largeartery atherosclerosis nor cardio-embolic etiology was found in the 10 cases of ischemic cerebrovascular events. Similarly, when evaluating the aPL positivity profiles in these two distinct groups, we found a significantly higher rate of antiB2GPI antibodies $(\operatorname{IgG} / \operatorname{IgM})$ in patients with stroke (Chi square test: $\mathrm{p}<0.05$ ). However, when focusing on the so-called triple positivity, we failed to observe any statistical difference (Table 2).

Significantly higher aGAPSS values were seen in patients with stroke [mean aGAPSS 8.9 (S.D. 4.7) Vs. mean aGAPSS 6.4 (S.D. 2.5); T test p:<0.05].

No statistical significant differences were observed when comparing separately each cardiovascular risk factor (i.e. smoking, diabetes, arterial hypertension) nor other thrombotic risk factors (hormonal therapy, immobilization, surgery or malignancy). 
Due to previous thrombosis, 25 patients were receiving oral anticoagulation with vitamin-K antagonists (VKA). Of those 25 patients, 20 patients suffered previous DVT and were treated with VKA with a target INR:2-3; Out of five patients with previous arterial thrombosis, three were treated with VKA with a target INR of 2-3 plus LDA, and two patients with VKA and a target INR between3-4. Moreover, three patients were treated with low molecular weight heparin (LMWH) (two patients because of pregnancy and one patient for secondary thromboprophylaxis whilst treated with chemotherapy for breast cancer). Eight patients with a previous history of obstetric APS were receiving anti-platelets agents.

Out of the 10 patients in whom the suspected stroke was confirmed, six had a history of previous venous thrombosis and were receiving VKA, INR target 2-3, one patient had a history of an arterial event and was treated with VKA INR 2-3 plus low dose aspirin (LDA) and one patient had a history of previous pregnancy complication and received LDA. For patients receiving therapy with VKA, the mean time in therapeutic range (TTR) calculated over the previous six months before the new cerebrovascular event was 77.7 (S.D. 6.6).

A tight control of cardiovascular risk factors and treatment implementation with statins and/or anti-hypertensive agent was ensured for all the patients and monitored by the treating physician's judgment. Therapeutic goals were set 
according to current recommendations [16,17]. 3.3. Evaluation at 12 Months of follow-up

During the 12 months of follow-up, no patient developed new episodes clinically suggestive of cerebral ischemia. In the non-stroke group, no changes at the follow-up MRI were observed when compared to baseline investigation.

After the diagnosis of stroke, $9 / 10$ patients were started/continued on anticoagulation therapy with VKA INR 2-3 plus LDA. The patient with previous arterial event was continued on VKA, increasing the INR target to 3-4. In agreement with the stroke unit team, one patient (with previous history of obstetric APS and subsequent stroke) was initially treated with VKA plus LDA but had to be switched to dual anti-platelet agents (LDA+Clopidogrel) after three months of VKA treatment because of unsatisfactory TTR, poor compliance and adherence on medical appointments.

After 12 months of follow-up, the 9 patients on VKA treatment showed no sign of cerebral progression based on new brain MRI. VKA was well tolerated and no adverse event was reported.

The one patient only treated with anti-platelet agents presented progression of the cerebral lesions as assessed after multidisciplinary panel evaluation. More specifically, at the brain MRI exam, a significant progression of the previously known lacunar infarctions involving the posterior limb of the left internal capsule/lateral thalamus and left frontal subcortical white matter was observed. 
Other cardiovascular risk factors were managed according to treating physician's judgment. 4.1 Discussion:

This prospective multicenter study assesses predictive factors for stroke in a cohort of 36 PAPS patients presenting with the onset of signs and/or symptoms of new focal neurological deficits suggestive of an acute stroke.

We found that ten patients (28\%) had a confirmed diagnosis of acute ischaemic cerebrovascular event. When stratifying patients for age, we found that $6 / 10$ patients with a documented stroke at the brain MRI scan belonged to the younger subgroup of patients $(<50$ years old $)$. Moreover, patients who experienced a stroke were receiving treatment according to the current recommendations. TTR for those patients treated with VKA as secondary thromboprophylaxis was $77.7 \%$.

Our results highlight the fact that despite being treated with anticoagulation (VKA treatment), patients with thrombotic PAPS still represent a sub-group of patients at high risk for thrombosis recurrences.

The identification of patients with aPL, who are at greater risk for developing any thrombotic event, i.e. especially arterial cerebral thrombosis, is still an unmet need. Therefore, risk stratifying in these patients remains crucial in this very setting.

In our prospective study, we demonstrate the clinical relevance of the aGAPSS for risk stratification in PAPS patients with stroke. In fact, significantly higher 
values of aGAPSS were found in patients with stroke. Those results are in line with a very recent study from our group, showing higher aGAPSS values in patients who experienced acute myocardial infarction when compared to those with other thrombosis[18].

Recently, a variety of prediction models for cardiovascular disease have been developed mainly focusing on stroke or ischaemic heart events. Among others, Hajifathalian and colleagues [19] elaborated a well thought out cardiovascular disease risk equation (Globorisk), adjusted for country origin, with routinely available information. Their risk score is based primarily on age, country of origin, arterial hypertension, smoking, and diabetes. Although promising, such score does not seem applicable to patients with autoimmune disorders, especially a rare disorder such as PAPS. As a matter of fact, in our cohort 15 patients out of 36 (42\%) were younger than 40 years old and the Globoriskcan therefore not be applied. Furthermore, just a portion of patients had diabetes ( 2 out of $36 ; 6 \%$ ) and/or arterial hypertension ( 9 out of $36 ; 25 \%$ ).

While anti-platelet agents are the gold standard therapies in stroke, patients with APS have been shown to benefit from long term anticoagulation [20]. Indeed, the only patient in our cohort who suffered with a progression of the ischaemic cerebral lesion at MRI, after 12 months follow up, was the one not treated with VKA.

When referring to the putative etiology of the stroke, patients were screened according treating physician's judgment and no significant causes rather than 
the presence of aPL and the described CV risk factors were identified. It was out of the scope of this study to assess the clinical accuracy of previously proposed systems for the etiological classification of ischemic stroke in patients with aPL (e.g. TOAST, CCS and ASCO systems [21-23]) . However, patients with confirmed events could be categorized as having "acute stroke of other determined etiology" according to the TOAST classification [21], as this category includes patients with more rare causes of stroke, such as non-atherosclerotic vasculopathies or hypercoagulable states, or hematologic disorders.

This study presents some limitations. First, the sample size is small, mainly due to the low prevalence of PAPS in the general population. Secondly, anticoagulant treatments before the cerebrovascular event and other cardiovascular risk factors treatment was based on the treating physician's judgment. Thirdly, previous medical history of PAPS patients was heterogeneous, including venous and arterial thrombosis and obstetric APS.

\section{Summary}

In summary, we think this study, acknowledging its limitations, contains some important clinical messages: PAPS is a thrombophilia with a high rate of recurrences; cerebrovascular events such as stroke affect a younger population when compared with non-APS population; a risk assessment, using appropriate tools such as aGAPSS, should be implemented to identify those patients at a higher risk of recurrences and have a strict control of all modifiable risk factors for cardiovascular events. 


\subsection{References}

1 Miyakis S, Lockshin MD, Atsumi T, et al. International consensus statement on an update of the classification criteria for definite antiphospholipid syndrome (APS). J Thromb Haemost 2006;4:295-306. doi:10.1111/j.15387836.2006.01753.x

2 Muscal E, Brey RL. Neurologic manifestations of the antiphospholipid syndrome: integrating molecular and clinical lessons. Curr Rheumatol Rep 2008;10:67-73.http://www.ncbi.nlm.nih.gov/pubmed/18457615 (accessed 21 Mar2017).

3 Cervera R, Piette J-C, Font J, et al. Antiphospholipid syndrome: clinical and immunologic manifestations and patterns of disease expression in a cohort of 1,000 patients. Arthritis Rheum 2002;46:1019-

27.http://www.ncbi.nlm.nih.gov/pubmed/11953980 (accessed 21 Mar2017).

4 de Amorim LCD, Maia FM, Rodrigues CEM. Stroke in systemic lupus erythematosus and antiphospholipid syndrome: risk factors, clinical manifestations, neuroimaging, and treatment. Lupus 2017;26:529-36. doi:10.1177/0961203316688784

5 Sciascia S, Sanna G, Khamashta MA, et al. The estimated frequency of antiphospholipid antibodies in young adults with cerebrovascular events: a 
systematic review. Ann Rheum Dis 2015;74:2028-33.

doi:10.1136/annrheumdis-2014-205663

6 Sciascia S, Sanna G, Murru V, et al. GAPSS: the Global Anti-Phospholipid Syndrome Score. Rheumatology (Oxford) 2013;52:1397-403. doi:10.1093/rheumatology/kes388

7 Stroke and transient ischaemic attack in over 16s: diagnosis and initial management | Guidance and guidelines | NICE. https://www.nice.org.uk/guidance/cg68/chapter/1-Guidance\#rapidrecognition-of-symptoms-and-diagnosis (accessed 1 Jun2017).

8 D'Agostino RB, Vasan RS, Pencina MJ, et al. General Cardiovascular Risk Profile for Use in Primary Care: The Framingham Heart Study. Circulation 2008;117:743-53. doi:10.1161/CIRCULATIONAHA.107.699579

9 Harris EN, Gharavi AE, Patel SP HG. Evaluation of the anti-cardiolipin antibody test: report of an international workshop held 4 April 1987. Clin Exp Immunol 1986;68:22.

10 Amengual 0, Atsumi T, Khamashta MA, et al. Specificity of ELISA for antibody to beta 2-glycoprotein I in patients with antiphospholipid syndrome. Br J Rheumatol 1996;35:123943.http://www.ncbi.nlm.nih.gov/pubmed/9010050 (accessed 22 Dec2016). 
11 Brandt JT, Triplett DA, Alving B, et al. Criteria for the diagnosis of lupus anticoagulants: an update. On behalf of the Subcommittee on Lupus Anticoagulant/Antiphospholipid Antibody of the Scientific and Standardisation Committee of the ISTH. Thromb Haemost 1995;74:118590.http://www.ncbi.nlm.nih.gov/pubmed/8560433 (accessed 22 Dec2016).

12 PENGO V, TRIPODI A, REBER G, et al. Update of the guidelines for lupus anticoagulant detection. J Thromb Haemost 2009;7:1737-40. doi:10.1111/j.1538-7836.2009.03555.x

13 Sciascia S, Sanna G, Murru V, et al. The global anti-phospholipid syndrome score in primary APS. Rheumatology (Oxford) 2015;54:134-8. doi:10.1093/rheumatology/keu307

14 Sciascia S, Cuadrado MJ, Sanna G, et al. Thrombotic risk assessment in systemic lupus erythematosus: validation of the global antiphospholipid syndrome score in a prospective cohort. Arthritis Care Res (Hoboken) 2014;66:1915-20. doi:10.1002/acr.22388

15 Sciascia S, Sanna G, Murru V, et al. The global anti-phospholipid syndrome score in primary APS. Rheumatol (United Kingdom) 2014;54. doi:10.1093/rheumatology/keu307

16 Stone NJ, Robinson JG, Lichtenstein AH, et al. 2013 ACC/AHA guideline on 
the treatment of blood cholesterol to reduce atherosclerotic cardiovascular risk in adults: a report of the American College of Cardiology/American Heart Association Task Force on Practice Guidelines. J Am Coll Cardiol 2014;63:2889-934. doi:10.1016/j.jacc.2013.11.002

17 ESH/ESC Task Force for the Management of Arterial Hypertension G, Fagard R, Narkiewicz K, et al. 2013 Practice guidelines for the management of arterial hypertension of the European Society of Hypertension (ESH) and the European Society of Cardiology (ESC): ESH/ESC Task Force for the Management of Arterial Hypertension. J Hypertens 2013;31:1925-38. doi:10.1097/HJH.0b013e328364ca4c

18 Radin M, Schreiber K, Costanzo P, et al. The adjusted Global AntiphosPholipid Syndrome Score (aGAPSS) for risk stratification in young APS patients with acute myocardial infarction. Int J Cardiol Published Online First: March 2017. doi:10.1016/j.ijcard.2017.02.155

19 Hajifathalian K, Ueda P, Lu Y, et al. A novel risk score to predict cardiovascular disease risk in national populations (Globorisk): a pooled analysis of prospective cohorts and health examination surveys. Lancet Diabetes Endocrinol 2015;3:339-55. doi:10.1016/S2213-8587(15)00081-9

20 Khamashta MA, Cuadrado MJ, Mujic F, et al. The management of thrombosis in the antiphospholipid-antibody syndrome. N Engl J Med 
1995;332:993-7. doi:10.1056/NEJM199504133321504

21 Adams HP, Bendixen BH, Kappelle LJ, et al. Classification of subtype of acute ischemic stroke. Definitions for use in a multicenter clinical trial. TOAST. Trial of Org 10172 in Acute Stroke Treatment. Stroke 1993;24:3541. doi:10.1161/01.STR.24.1.35

22 Ay H, Benner T, Murat Arsava E, et al. A Computerized Algorithm for Etiologic Classification of Ischemic Stroke The Causative Classification of Stroke System. doi:10.1161/STROKEAHA.107.490896

23 Amarenco P, Bogousslavsky J, Caplan LR, et al. New Approach to Stroke Subtyping: The A-S-C-O (Phenotypic) Classification of Stroke. Cerebrovasc Dis 2009;27:502-8. doi:10.1159/000210433 
Acknowledgments: None

Disclosure of Conflicts of Interest: None declared

Funding: None declared 


\section{Legend of Tables and Figures:}

Table 1. Demographic, clinical and laboratory characteristics of the cohort

Table 2. Patients cardiovascular risk factors and aPL positivity between groups 
